\title{
Indirubin-3'-oxime induces mitochondrial dysfunction and triggers growth inhibition and cell cycle arrest in human neuroblastoma cells
}

\author{
XUE-MEI LIAO and KWOK-NAM LEUNG \\ Biochemistry Programme, School of Life Sciences, The Chinese University of Hong Kong, \\ Hong Kong, SAR, P.R. China
}

Received July 31, 2012; Accepted September 20, 2012

DOI: $10.3892 /$ or.2012.2094

\begin{abstract}
Neuroblastoma is the most common extracranial solid tumor found in infancy and childhood. Current multimodal therapies such as surgery, chemotherapy, radiotherapy and stem cell transplantation often cause inevitable severe side-effects, therefore, it is necessary to develop novel drugs with higher efficacy on neuroblastoma cells and minimal side-effects on normal cells. Indirubin-3'-oxime (I3M), an indigo alkaloid, was found to exhibit potent antitumor activities on various types of cancer cells. However, its modulatory effects on human neuroblastoma and the underlying mechanisms remain poorly understood. As mitochondrial biogenesis and function play critical roles in cell growth and survival, in the present study the effects of I3M on mitochondrial functions and their correlation to the anticancer effect of I3M on human neuroblastoma cells were investigated. I3M was found to inhibit the growth of the human neuroblastoma LA-N-1, SH-SY5Y and SK-N-DZ cells in a dose- and time-dependent manner, but exhibited little, if any, direct cytotoxicity on normal cells. Mechanistic studies showed that I3M specifically decreased the expression of the mitochondrial regulators ERR $\gamma$ and PGC- $1 \beta$ and resulted in decreased mitochondrial mass and altered mitochondrial function characterized by a reduction in mitochondrial membrane potential and elevation of reactive oxygen species levels in LA-N-1 cells. I3M also increased the level of CDK inhibitor $\mathrm{p} 27^{\mathrm{Kip} 1}$ and reduced the levels of CDK2 and cyclin E in LA-N-1 cells, leading to cell cycle arrest at the G0/G1 phase. Collectively, these results suggest that mitochondrial dysfunction might be an important mechanism underlying the I3M-induced cell cycle arrest.
\end{abstract}

Correspondence to: Professor Kwok-Nam Leung, Biochemistry Programme, School of Life Sciences, The Chinese University of Hong Kong, Shatin, Hong Kong, SAR, P.R. China

E-mail:knleung@cuhk.edu.hk

Key words: indirubin-3'-oxime, cell cycle arrest, human neuroblastoma, mitochondrial dysfunction, reactive oxygen species

\section{Introduction}

Neuroblastoma, a malignant neoplasm of the sympathetic nervous system, is thought to arise from the neural crest cells (1). It is the most common and fatal extracranial solid tumor in childhood and accounts for 8-10\% of all childhood cancers. Children with localized neuroblastoma can be cured with surgery and/or chemotherapy. Approximately 50\% of neuroblastoma patients have high-risk disease with widespread tumor dissemination. For these children, current treatment modalities consist of intensive chemotherapy, surgery, radiotherapy, stem cell transplantation, differentiation therapy and immunotherapy (2). However, these treatments often cause severe and inevitable side-effects and relapse frequently occurs in high-risk patients despite aggressive multimodal therapy. Neuroblastoma currently accounts for $\sim 15 \%$ of childhood cancer-related mortality rates and is a major problem in pediatric oncology (2). It is important to develop novel drugs which are potent in inhibiting the proliferation of cancer cells while exhibiting minimal cytotoxicity towards the normal cells. The use of new promising therapeutic compounds derived from natural products or Chinese herbs has attracted much interest as an alternative in cancer treatment.

Indirubin, a 3, 2'-bisindole isomer of indigo, is an active ingredient of Danggui Longhui Wan used in Traditional Chinese Medicine for the treatment of chronic diseases such as chronic myelogenous leukemia (3). In the past decade, a number of indirubin analogs have been synthesized to optimize this promising drug scaffold. Indirubin-3'-oxime (I3M) (Fig. 1) is a cell-permeable derivative of indirubin commercially available with increased kinase inhibitory effects (4) and increased solubility (5). Previous studies identified indirubin and $\mathrm{I} 3 \mathrm{M}$ as potent inhibitors of cyclin-dependent kinases

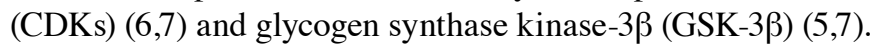
I3M has been reported to inhibit cell proliferation and arrest the cell cycle progression of various cancer cells $(6,8,9)$. It was also shown to induce apoptosis in human cervical cancer HeLa cells, colon cancer HCT116 cells, hepatoma HepG2 cells and renal cell cancer cell lines in a time- and dose-dependent manner $(10,11)$. Aside from the induction of apoptosis, I3M was found to induce necrosis in nocodazole-synchronized SV-40 transformed human breast epithelial HBL-100 cells 
(9). Previously, I3M was reported to exhibit antitumor effects against benzo( $\alpha$ ) pyrene-induced lung cancer in A/J mice through its apoptotic action (12). However, the modulatory effects and the detailed molecular action mechanisms of I3M on human neuroblastoma cells have yet to be studied.

Functional mitochondria act as a primary source of energy output and are required for all cellular activities. It is known that mitochondria are involved in a wide range of cellular processes, such as cell signaling, cellular differentiation, cell death, as well as the control of the cell cycle and cell growth (13). Recent studies revealed that mitochondrial biogenesis and function are intimately linked to cell cycle progression. For instance, knockdown of human mitochondrial transcription termination factor 4 (hMTERF4) in human cervical carcinoma HeLa cells induced accumulation of sub-G1 phase cells, whereas its overexpression promoted cell proliferation (14). In a series of experiments expanding on this theme in Drosophila, it was demonstrated that mutation in the cytochrome oxidase subunit Va led to a reduction in ATP production in the cell and arrested it in the G1 phase (15), and loss of function of Pdsw, a subunit of mitochondrial complex I, led to increased reactive oxygen species (ROS) production and blocked the cell in G1-S transition through reduction in cyclin E protein and upregulation of the CDK inhibitor p27 $7^{\mathrm{Kip} 1}$ protein (16). The constitutively active nuclear hormone receptors, estrogenrelated receptor $\alpha$ and $\gamma(E R R \alpha$ and ERR $\gamma)$, together with their co-activators, the peroxisome proliferator-activated receptor- $\gamma$ (PPAR $\gamma$ ) co-activator- $1 \alpha$ and $-1 \beta$ (PGC- $1 \alpha$ and PGC- $1 \beta$ ), are thought to regulate mitochondrial biogenesis and oxidative phosphorylation (17); they govern several important aspects of mitochondrial function (18).

In this study, we hypothesized that I3M might induce cell cycle arrest in the human neuroblastoma LA-N-1 cells which might be mediated through the triggering of mitochondrial dysfunction. Our present findings clearly show that I3M altered mitochondrial biogenesis and function, and finally resulted in cell cycle arrest in human neuroblastoma LA-N-1 cells at the G0/G1 phase.

\section{Materials and methods}

Cell culture and reagents. Human neuroblastoma cell line LA-N-1 was purchased from RIKEN BioResource Center Cell Bank (Japan), while SH-SY5Y, SK-N-DZ, human embryonic kidney HEK-293 and human hepatocyte-like WRL-68 cell lines were purchased from the American Type Culture Collection (ATCC, USA). LA-N-1 cells were cultured in RPMI-1640 medium (Gibco, USA) supplemented with 10\% fetal bovine serum (FBS; Gibco), $1 \%$ antibiotics $(100 \mathrm{U} / \mathrm{ml}$ penicillin G, $100 \mu \mathrm{g} / \mathrm{ml}$ streptomycin sulfate and $0.25 \mu \mathrm{g} / \mathrm{ml}$ amphotericin B in $0.85 \%$ saline). SH-SY5Y cells were grown in DMEM/F12 medium (Gibco) supplemented with 15\% FBS, $1 \%$ antibiotics, $1 \mathrm{mM}$ sodium pyruvate and $1 \mathrm{mM}$ MEM with non-essential amino acids (NEAA) solution. SK-N-DZ and HEK 293 cells were cultured in DMEM medium supplemented with $10 \%$ FBS and $1 \%$ antibiotics. WRL-68 cells were cultured in MEM medium (Gibco) supplemented with 10\% FBS and 1\% antibiotics. The primary embryonic cortical neurons from SD rats were kindly provided by Professor K.F. Lau, School of Life Sciences, The Chinese University of Hong Kong. The primary

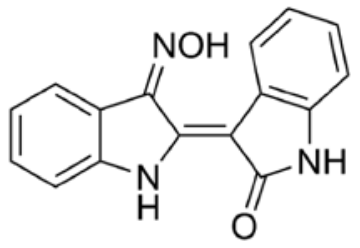

Figure 1. Chemical structure of indirubin-3'-oxime.

embryonic cortical neurons were cultured in MEM medium supplemented with 5\% FBS, glucose $(18 \mathrm{mM})$, L-glutamine $(2 \mathrm{mM})$, insulin $(5 \mu \mathrm{g} / \mathrm{ml})$, progesterone $(0.02 \mu \mathrm{M})$, putrescine $(100 \mu \mathrm{M})$, selenium $(30 \mathrm{pM}), \beta$-mercaptoethanol $(25 \mu \mathrm{M})$ and $1 \%$ antibiotics. All cell lines were cultured at $37^{\circ} \mathrm{C}$ in a humidified incubator supplied with $5 \% \mathrm{CO}_{2} . \mathrm{I} 3 \mathrm{M}$ was purchased from Sigma-Aldrich (USA). Stock solution of I3M (10 mM) was prepared by dissolving the powdered form of I3M (with a molecular weight of 277.28 and $>98 \%$ purity) in $100 \%$ dimethyl sulfoxide (DMSO; Sigma, USA) and stored in the dark at $-20^{\circ} \mathrm{C}$ until use. All other chemicals were purchased from Sigma unless otherwise stated.

Cell growth assay. Cell growth was measured using both the colorimetric MTT assay and fluorometric cell proliferation assay. Cells were seeded in 96-well plates, cultured overnight and then treated with different concentrations of I3M. Following treatment for the indicated periods of time, cell growth was measured using the MTT assay and recorded by a Benchmark microplate reader (Bio-Rad Laboratories, USA) as previously described (19). In addition, cell proliferation was measured using the CyQUANT ${ }^{\circledR}$ NF Cell Proliferation Assay kit (Molecular Probes, Invitrogen Corp., USA) and recorded by a fluorescence plate reader (Tecan Polarion, USA) (20).

Colony-forming assay. Cell colony-forming ability was determined as previously described (21). Briefly, the 6-well tissue culture plates were pretreated with poly-D-lysine hydrobromide for $4 \mathrm{~h}$ and washed once with deionized water. Then, 400 cells were added into each well of a 6-well plate and allowed to settle overnight. Cells were treated with I3M for $24 \mathrm{~h}$. On the following day, the medium was replaced with completed RPMI medium. The medium was changed every 3 days. After 6 days, colonies were fixed with methanol and stained with Hemacolor staining solutions. Colonies were counted and the percentage of colonies relative to solventtreated control was calculated.

Apoptosis assay. The Cell Death Detection ELISA ${ }^{\text {PLus }}$ kit (Roche Applied Science, Switzerland) was used to quantify the enrichment of cytoplasmic nucleosomes which reflects the induction of apoptosis in the cells. LA-N-1 cells were seeded in 96-well plates at a density of $1.5 \times 10^{4}$ cells per well. On the following day, cells were treated with solvent control (0.1\% DMSO) or different concentrations of I3M for $48 \mathrm{~h}$. Doxorubicin $(0.2 \mu \mathrm{M})$ was used as positive control. Procedures were performed according to the manufacturer's instructions and absorbance was measured at $405 \mathrm{~nm}$ with reference to $490 \mathrm{~nm}$ by a Benchmark microtiter plate reader (Bio-Rad Laboratories) after color development, which was proportional 
to the amount of nucleosomes present in the samples. The results were expressed as enrichment factor which represents the relative levels of mono- and oligo-nucleosomes compared with the solvent control.

Cell cycle analysis. LA-N-1 cells were seeded in $60-\mathrm{mm}$ dishes at a density of $9 \times 10^{5}$ cells per dish and incubated overnight. After synchronizing in plain RPMI medium for $24 \mathrm{~h}$, cells were treated with different concentrations of I3M for 48 h. Following treatment, cells were fixed with $70 \%$ ethanol, treated with $50 \mu \mathrm{g} / \mathrm{ml}$ RNase A (Sigma), stained with $40 \mu \mathrm{g} / \mathrm{ml}$ propidium iodide (Sigma) and analyzed by the FACSCanto flow cytometer (BD Biosciences, USA) for DNA synthesis and cell cycle status. The percentages of cells in the G0/G1, S and G2/M cell cycle phases were calculated by the ModFit 3.0 program (BD Biosciences).

Quantitative real-time PCR. Total RNA from LA-N-1 cells was extracted by TRIzol reagent (Invitrogen) according to the manufacturer's protocol. The first-strand cDNA was generated with random primers (Invitrogen) by M-MLV reverse transcription kits (Invitrogen). Quantitative real-time PCR analysis was performed with SYBR premix Ex Taq kit (Takara, China) using ABI-7500 Real-Time PCR System (Applied Biosystems, USA). Relative gene expression was normalized to $\beta$-actin level. The sequences of primers used are listed in Table I.

Western blot analysis. Cell pellets were collected at the indicated time points and total proteins were extracted by cell lysis buffer. Protein concentration was measured by the Bradford reagent (Sigma). Protein extracts were analyzed by $10 \%$ sodium dodecyl sulphate polyacrylamide gel electrophoresis and blotted onto polyvinylidene fluoride membranes. Membranes were blocked with 5\% non-fat dairy milk in Tris-buffered saline (20 mM Tris, $150 \mathrm{mM} \mathrm{NaCl}, \mathrm{pH} 7.4$ ) with $0.05 \%$ Tween-20 and incubated with mouse anti-human CDK2 antibody (Cell Signaling Technology, USA), rabbit anti-human-ERR $\alpha,-E R R \gamma,-P G C-1 \alpha$ and -PGC-1 $\beta$ antibodies (Cell Signaling Technology), rabbit anti-human cyclin E antibody (Santa Cruz Biotechnology, USA), or mouse antihuman $\beta$-actin antibody (Sigma) followed by HRP-conjugated secondary antibody (GE Healthcare UK Ltd., UK) and developed with the ECL reagent (Santa Cruz Biotechnology).

Mitochondrial mass assay. Mitotracker Green (Invitrogen) was added and quantified as described by Liao et al (22). Briefly, I3M-treated cells were incubated in serum-free medium (pre-warmed to $37^{\circ} \mathrm{C}$ ) containing $150 \mathrm{nM}$ Mitotracker Green FM for $20 \mathrm{~min}$ in the dark. After staining, the cells were washed twice with cold PBS and suspended in $500 \mu 1$ PBS. Subsequently, cells were analyzed on a FACSCanto flow cytometer with excitation at $488 \mathrm{~nm}$ and emission at $516 \mathrm{~nm}$. Data were processed using the CellQuest program (BD Biosciences).

Reactive oxygen species assay. ROS production in mitochondria was measured by a cell-permeable fluorogenic probe MitoSOX ${ }^{\mathrm{TM}}$ Red (Invitrogen) that is selectively targeted to the mitochondria, where it specifically reacts with superoxide
Table I. Primer sequences used for RT-PCR based on human genes and shown from 5'- to 3'-.

$\begin{array}{ll}\beta \text {-actin-forward } & \text { CAGGAGATGGCCACTGCCGCA } \\ \beta \text {-actin-reverse } & \text { CTCCTTCTGCATCCTGTCAGCA } \\ \text { CDK2-forward } & \text { TTGGGCTATTTGGACTCAGG } \\ \text { CDK2- reverse } & \text { AAGGGTGGTGGAGGCTAACT } \\ \text { Cyclin E-forward } & \text { CGTGCGTTTGCTTTTACAGA } \\ \text { Cyclin E- reverse } & \text { CTGGAGGTGGCTGGTGTACT } \\ \text { ERR } \gamma \text {-forward } & \text { AATGAATGTGAAAGGCTGTG } \\ \text { ERR } \gamma \text {-reverse } & \text { TAGTTGTGTGTGAATGTATGGG } \\ \text { PGC-1 } \beta \text {-forward } & \text { CTCATACGCTACATGCACAC } \\ \text { PGC-1 } \beta \text {-reverse } & \text { GCCAAGACCAGCAGCTC } \\ \text { ATP5b-forward } & \text { TGTTGGCAGTGAGCATTACG } \\ \text { ATP5b-reverse } & \text { ACCTGTGAAGACCTCAGCAAC } \\ \text { Tfam-forward } & \text { AAGATTCCAAGAAGCTAAGGGTGA } \\ \text { Tfam-reverse } & \text { CAGAGTCAGACAGATTTTTCAGTTT } \\ \text { SOD1-forward } & \text { ATTCTGTGATCTCACTCTCAGG } \\ \text { SOD1-reverse } & \text { TCGCGACTAACAATCAAAGT } \\ \text { SOD2-forward } & \text { GGGGCACGTGTTAAGGATGT } \\ \text { SOD2-reverse } & \text { AGAATCTTGAGTTTCCTTCACCG }\end{array}$

anion as previously described (22). Briefly, I3M-treated cells were loaded with $5 \mu \mathrm{M}$ MitoSOX Red for $10 \mathrm{~min}$ at $37^{\circ} \mathrm{C}$, washed with PBS, and the fluorescence was detected with the FACSCanto flow cytometer with excitation at $488 \mathrm{~nm}$ and emission at $580 \mathrm{~nm}$. Data were processed using the CellQuest program.

Mitochondrial membrane potential determination. Mitochondrial membrane potential $\left(\Delta \Psi_{\mathrm{m}}\right)$ was analyzed by a fluorescent dye JC-1 (Invitrogen). JC-1 is capable of selectively entering mitochondria where it forms monomers and emits green fluorescence when $\Delta \Psi_{\mathrm{m}}$ is relatively low. At high $\Delta \Psi_{\mathrm{m}}, \mathrm{JC}-1$ aggregates and gives red fluorescence. Briefly, I3M-treated cells were incubated in serum-free medium (prewarmed to $37^{\circ} \mathrm{C}$ ) containing $4 \mu \mathrm{M} \mathrm{JC}-1$ for $20 \mathrm{~min}$ in the dark. After staining, the cells were washed once with cold PBS and suspended in $500 \mu \mathrm{l}$ PBS. Green $(525 \mathrm{~nm})$ and red $(590 \mathrm{~nm})$ fluorescence were detected on a FACSCanto flow cytometer. Data were processed by using the CellQuest program.

Statistical analysis. All assays were performed at least three times and the data are presented as the mean \pm SD. The Student's t-test was used to determine the significant difference between the drug-treated group and the control group. $\mathrm{P}<0.05$ is regarded as statistically significant. Asterisks indicate significant differences: ${ }^{*} \mathrm{P}<0.05,{ }^{* *} \mathrm{P}<0.01,{ }^{* * *} \mathrm{P}<0.001$.

\section{Results}

Indirubin-3'-oxime inhibits the growth and colony formation of human neuroblastoma cells in vitro. To examine the effect of I3M on the growth of human neuroblastoma cells, the MTT assay, CyQUANT NF cell proliferation assay and colony- 
A
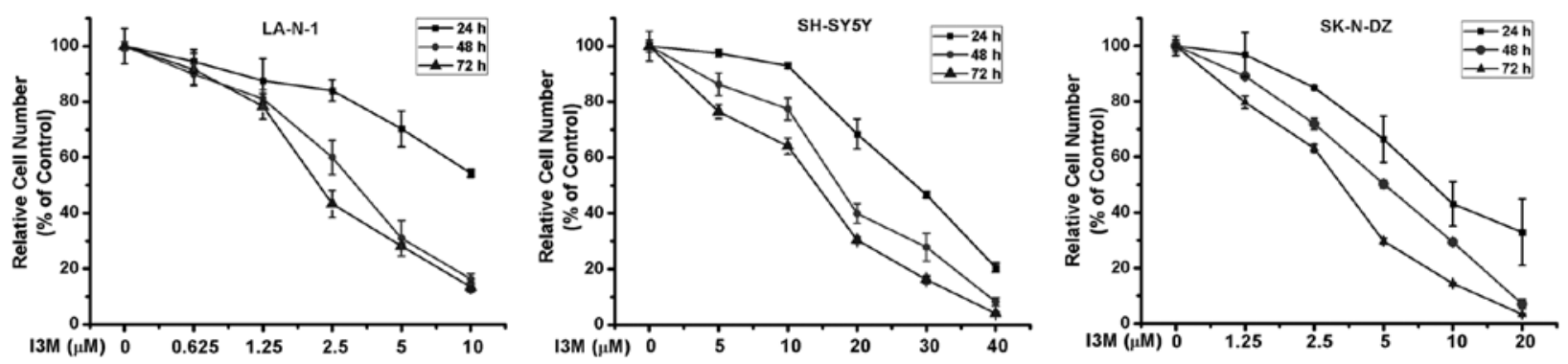

\section{B}
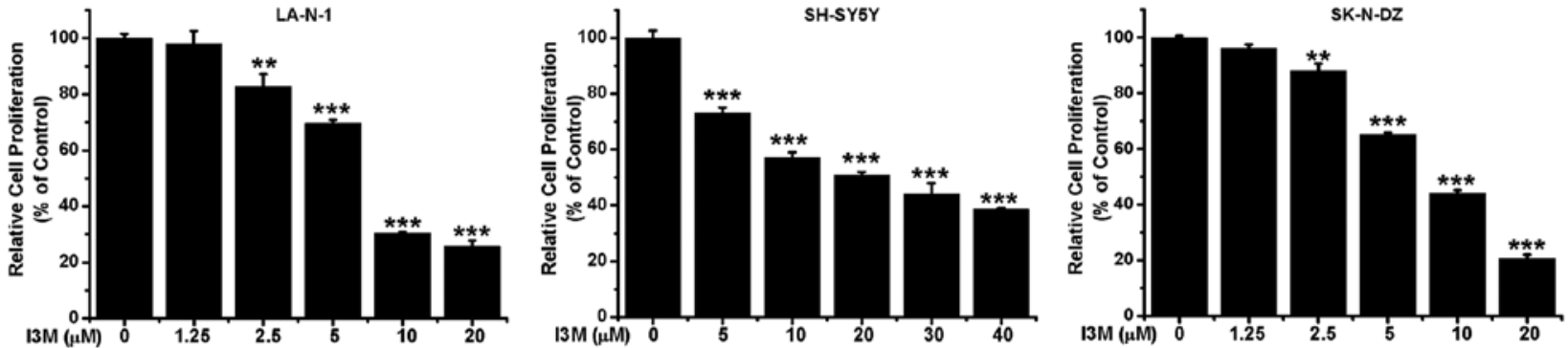

C

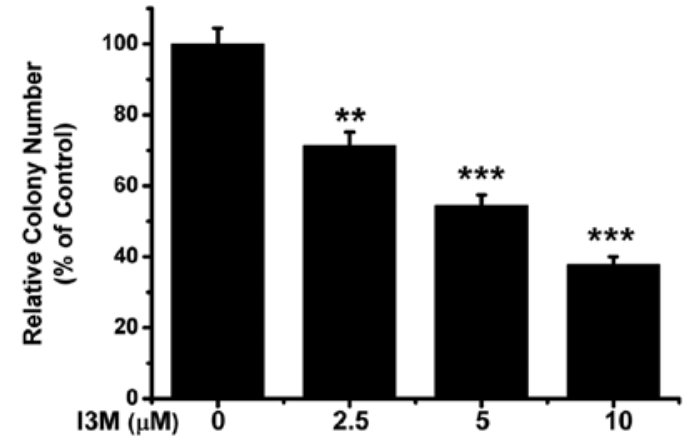

SD Rat Cortical Neurons

HEK-293 Cells
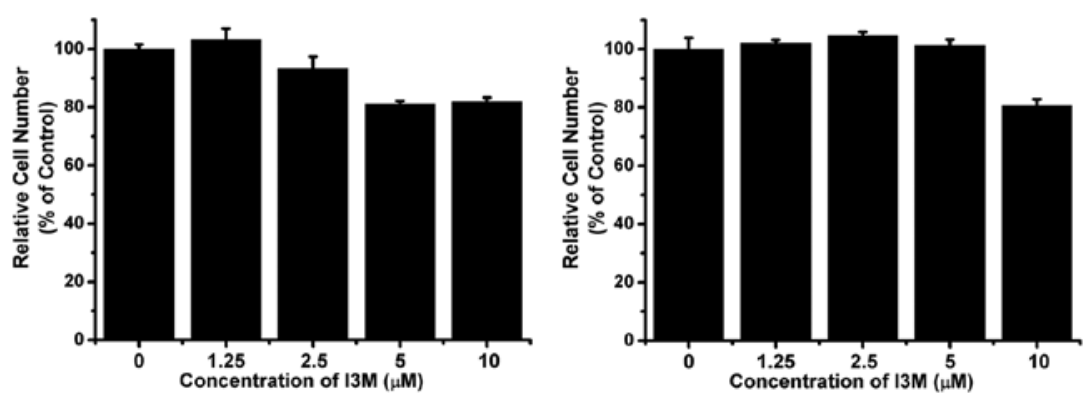

WRL-68 Cells

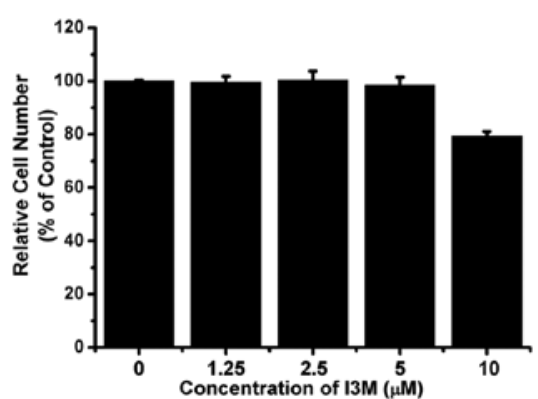

Figure 2. Growth-inhibitory effect of I3M on human neuroblastoma cells vs. normal cells. LA-N-1 cells $\left(1.5 \times 10^{4}\right.$ cells/well), SH-SY5Y cells ( $10^{4}$ cells/well) or SK-N-DZ cells $\left(10^{4}\right.$ cells/well) were treated with solvent control (0.1\% DMSO) or various concentrations of I3M. (A) After treatment for 24,48 or $72 \mathrm{~h}$, the relative cell number was measured by the MTT assay. (B) After treatment for $48 \mathrm{~h}$, relative cell proliferation was measured using the CyQUANT NF Cell Proliferation Assay kit. (C) LA-N-1 cells (400 cells/well) in 6-well plates were treated with I3M for $24 \mathrm{~h}$. After 6 days, colonies were fixed, counted and shown as percentage of solvent-treated control in 3 independent experiments. (D) Rat primary embryonic cortical neurons $\left(10^{6}\right.$ cells/well), human embryonic kidney HEK-293 cells $\left(8 \times 10^{3}\right.$ cells/well) or human hepatocyte-like cell line WRL-68 cells $\left(5 \times 10^{3}\right.$ cells/well) were treated with solvent control or various concentrations of I3M for $48 \mathrm{~h}$ and the relative cell number was determined by the MTT assay. Data are expressed as the means $\pm \mathrm{SD}$. ${ }^{* * *} \mathrm{P}<0.01 ;{ }^{* * *} \mathrm{P}<0.001$.

forming assay were performed. Three human neuroblastoma cell lines LA-N-1, SH-SY5Y and SK-N-DZ, were exposed to increasing concentrations of I3M for 24,48 and $72 \mathrm{~h}$ and the relative cell number was monitored by MTT assay. As shown in Fig. 2A, I3M dose- and time-dependently reduced the growth of the three cell lines. The $50 \%$ inhibitory concentration $\left(\mathrm{IC}_{50}\right.$ ) values of I3M on LA-N-1, SH-SY5Y and SK-N-DZ cells after 48 -h treatment were $3.27 \pm 0.9,18.15 \pm 1.91$ and $5.4 \pm 0.28 \mu \mathrm{M}$ respectively, indicating that $\mathrm{I} 3 \mathrm{M}$ has the highest potency on LA-N-1 cells. I3M also inhibited the proliferation of LA-N-1, SH-SY5Y and SK-N-DZ cells in a dose-dependent manner after 48-h treatment (Fig. 2B). It has been shown that the colony-forming ability of drug-treated tumor cells is related to their tumorigenicity in vivo (23). I3M was found to 


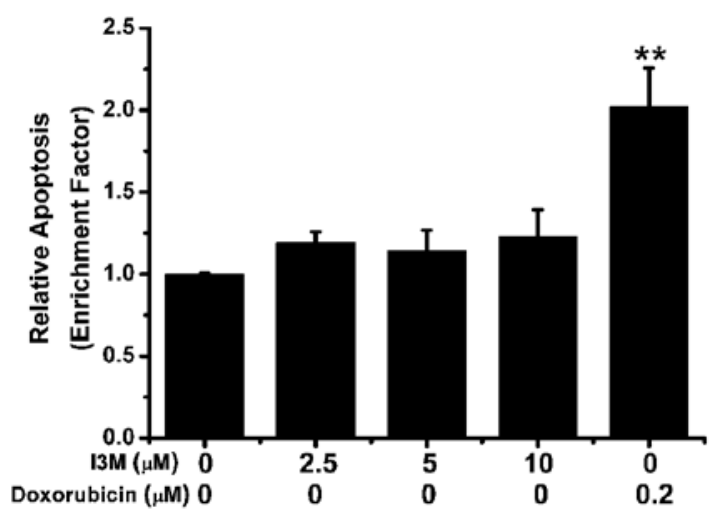

Figure 3. I3M does not induce apoptosis in LA-N-1 cells. LA-N-1 cells were seeded in 96-well plates at a density of $1.5 \times 10^{4}$ cells per well. On the following day, cells were treated with solvent control (0.1\% DMSO) or different concentrations of I3M for $48 \mathrm{~h}$. Doxorubicin $(0.2 \mu \mathrm{M})$ was used as positive control. The released cytoplasmic mono- and oligo-nucleosomes were quantified by the Cell Death Detection ELISA ${ }^{\text {PLus }}$ kit according to the manufacturer's instructions. Enrichment factor represents the relative levels of mono- and oligo-nucleosomes compared with solvent control. ${ }^{* *} \mathrm{P}<0.01$.

decrease the colony-forming ability of LA-N-1 cells dosedependently (Fig. 2C). A drug with a high therapeutic index indicates that it is potent in inhibiting the proliferation of cancer cells while exhibiting little or minimal cytotoxicity towards the normal cells. In this study, I3M was found to exhibit little, if any, direct cytotoxicity on the normal cells such as rat primary embryonic cortical neuronal cells, human embryonic kidney HEK-293 cells and human hepatocyte-like WRL-68 cells at or below $10 \mu \mathrm{M}$ concentrations (Fig. 2D).

Indirubin-3'-oxime induces G0/G1 cell cycle arrest in $L A-N-1$ cells. Since I3M caused a significant inhibition of cell growth in the human neuroblastoma LA-N-1 cells, the underlying mechanisms were investigated. The possible mechanisms of I3M leading to its potent anti-proliferative effect is its ability to induce apoptosis or interrupt the normal cell cycle progression. Initially, the Cell Death Detection ELISA $^{\text {PLUS }}$ kit was used to determine whether I3M could induce apoptosis in the neuroblastoma LA-N-1 cells. I3M failed to induce significant apoptosis of the LA-N-1 cells at concentrations that are inhibitory for the growth of the cells (Fig. 3). Next, we investigated whether the I3M-induced cell growth inhibition was due to an arrest at a specific point of the cell cycle. It was found that at concentrations $>2.5 \mu \mathrm{M}, \mathrm{I} 3 \mathrm{M}$ significantly induced cell cycle arrest at the G0/G1 phase, accompanied by a significant decrease in the percentage of cells in the $\mathrm{S}$ and G2/M phase (Fig. 4A). The effects of I3M on the expression of the G0/G1 phase regulatory proteins including cyclins, cyclin-dependent kinases (CDKs) and CDK inhibitors in LA-N-1 cells were then examined. As shown in Fig. 4B, the I3M-treated cells exhibited an increase in $\mathrm{p} 27^{\mathrm{Kip} 1}$, and a decrease in the protein levels of CDK2 and cyclin E compared to the corresponding untreated control. Using the technique of real-time polymerase chain reaction, it was shown that the mRNA levels of CDK2 and cyclin E decreased in a dose-dependent manner following treatment with I3M (Fig. 4C and D).
A
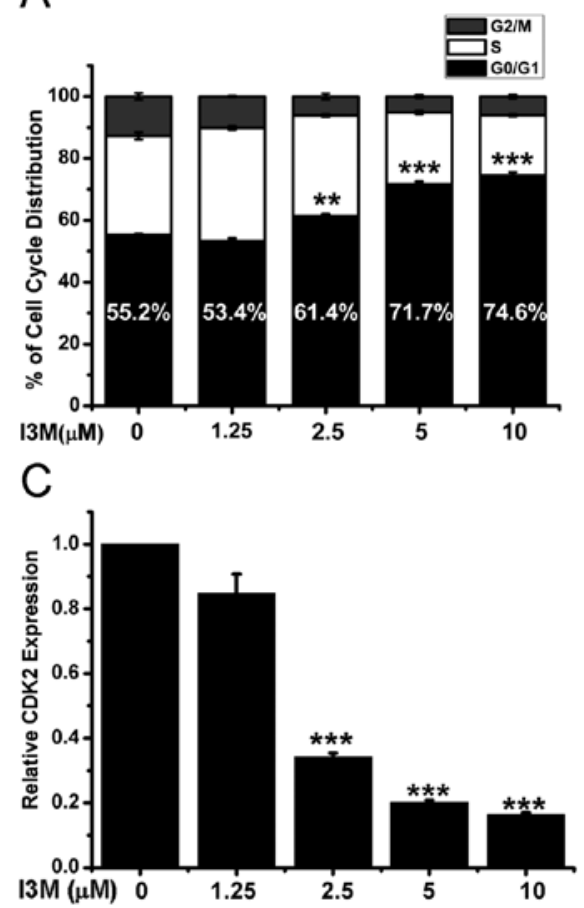

B

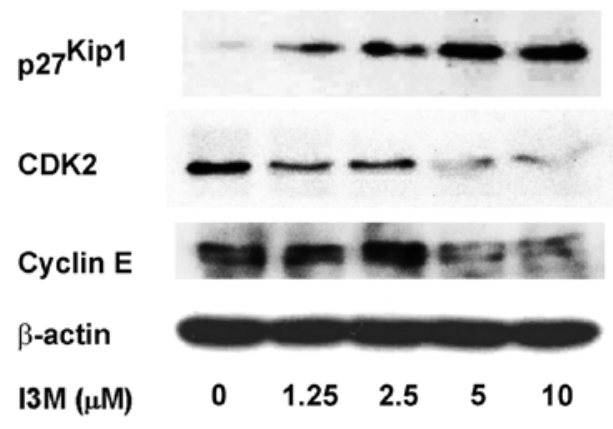

D

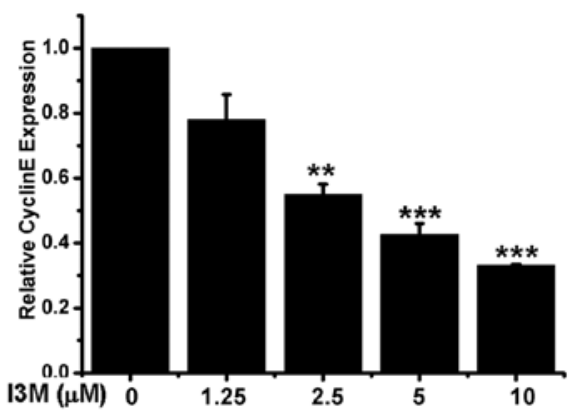

Figure 4. I3M induces cell cycle arrest at the G0/G1 phase in LA-N-1 cells. (A) LA-N-1 cells were seeded in 60-mm dishes (9x105 cells per dish) and incubated overnight. Cell cycle distribution of cells treated without or with I3M for $48 \mathrm{~h}$ was analyzed by flow cytometry using the ModFit program. (B) LA-N-1 cells were incubated either with solvent control (0.1\% DMSO) or with various concentrations of I3M for 48 h. Protein levels of p27 ${ }^{\mathrm{Kipl}}$, CDK2 and cyclin E were assayed by western blots with $\beta$-actin as an internal control. mRNA expression levels of CDK2 (C) and cyclin E (D) were detected using quantitative real-time PCR (qRT-PCR) after 24 h of I3M treatments. ${ }^{* *} \mathrm{P}<0.01 ;{ }^{* * * *} \mathrm{P}<0.001$. 
A

B
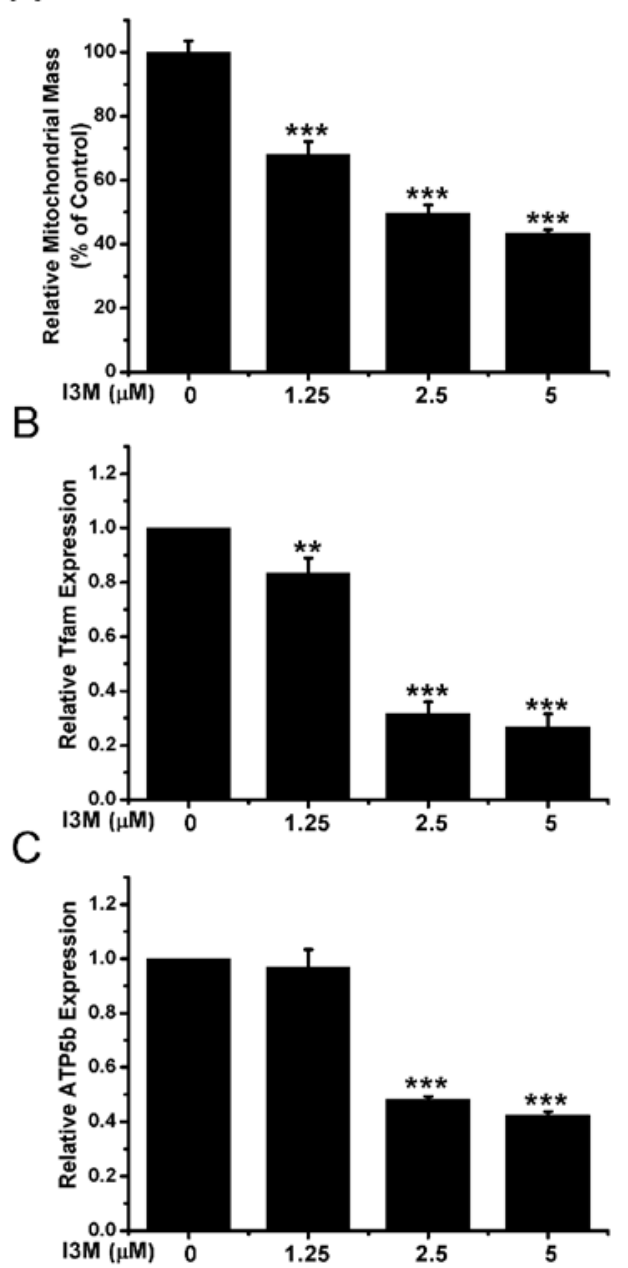

D

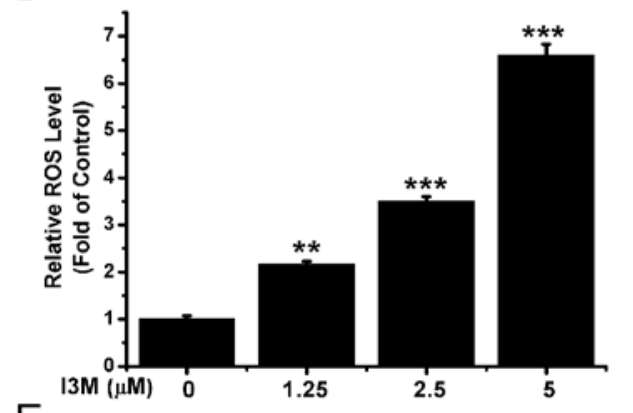

E

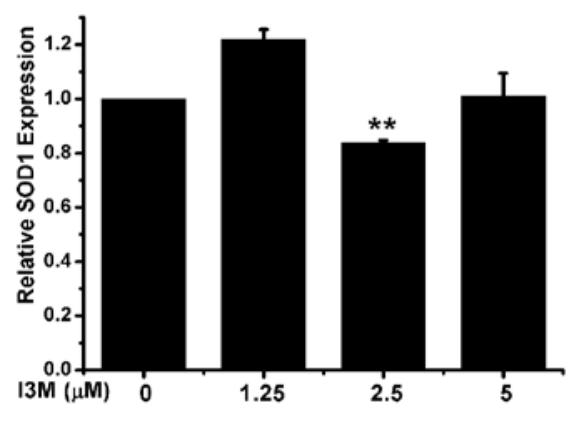

$\mathrm{F}$

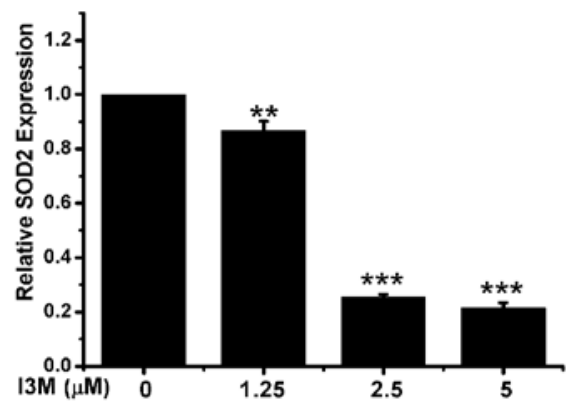

Figure 5. I3M reduces mitochondrial mass and elevated mitochondrial ROS in LA-N-1 cells. LA-N-1 cells were seeded in 24-well plates at a density of $1.5 \times 10^{5}$ cells per well and incubated overnight. (A) Mitochondrial mass of cells treated with I3M for $48 \mathrm{~h}$ were stained by Mitotracker Green and quantified. The amount of mitochondrial mass in the solvent-treated control was set as $100 \%$. (D) Mitochondrial ROS levels of cells treated with I3M for $48 \mathrm{~h}$ were stained by MitoSOX Red and quantified. The amount of mitochondrial ROS in the solvent-treated control was set as 1 . The mRNA expression levels of Tfam (B), ATP5b (C), SOD1 (E) and SOD2 (F) were detected using qRT-PCR after $24 \mathrm{~h}$ of I3M treatment. The relative level for the solvent-treated control was set as 1. ${ }^{* *} \mathrm{P}<0.01 ;{ }^{* * *} \mathrm{P}<0.001$.

Indirubin-3'-oxime causes mitochondrial dysfunction in $L A-N-1$ cells. The roles of mitochondrial biogenesis and function in cell growth and proliferation have been investigated. Mitochondrial function is known to play a pivotal role in the maintenance of cellular homeostasis. Previous studies have shown that mitochondrial dysfunction causes cell cycle arrest in neuronal PC12 cells (24). In the present study, whether the I3M-mediated growth suppression of LA-N-1 cells was correlated with mitochondrial biogenesis and function was examined. The changes in mitochondrial mass were measured by staining the cells with a fluorescent MitoTracker Green dye that stains the mitochondria independent of its $\Delta \Psi_{\mathrm{m}}$. It was found that I3M dose-dependently reduced the mitochondrial mass (Fig.5A). Correspondingly, the expression levels of mitochondrial transcription factor A (Tfam) and ATP synthase (ATP5b) that are involved in regulating mitochondrial mass and ATP production, respectively, were significantly reduced by I3M (Fig. 5B and C). It would be of interest to examine if I3M altered mitochondrial function in addition to reducing the mitochondrial mass in the human neuroblastoma LA-N-1 cells. First, the effect of I3M on the $\Delta \Psi_{\mathrm{m}}$ of LA-N-1 cells was studied. A fluorescent dye JC-1, which gives a red fluorescence when $\Delta \Psi_{\mathrm{m}}$ is high and green fluorescence when $\Delta \Psi_{\mathrm{m}}$ is low, was used to determine the overall electron transport chain activity. Indeed, quantification measurements indicated that I3M dose-dependently decreased the red to green fluorescence ratio, indicating a decrease in $\Delta \Psi_{\mathrm{m}}$ (Fig. 6). Decreased $\Delta \Psi_{\mathrm{m}}$ inhibits ATP generation, results in oxidative stress and leads to mitochondrial dysfunction. In order to demonstrate that $\mathrm{I} 3 \mathrm{M}$ could result in oxidative stress in LA-N-1 cells, the ability of I3M to induce the generation of ROS was examined. Using a fluorescent MitoSOX Red dye, which selectively targets the mitochondria and reacts with superoxide anion, it was found that I3M elevated mitochondrial ROS levels in LA-N-1 cells dose-dependently (Fig. 5D). An endogenous antioxidant system that protects cells against ROS is characterized by superoxide dismutases (SODs). We also checked the mRNA expression levels of SOD1 and SOD2 in I3M-treated cells and found that it selectively suppressed the expression of SOD2, which resides in the mitochondria, but not SOD1 that is found mainly in intracellular cytoplasmic spaces (25) (Fig. 5E and F). 
A

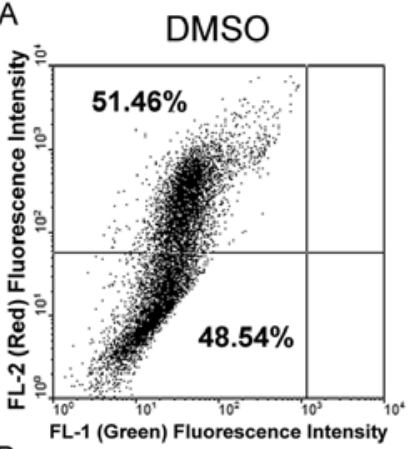

B

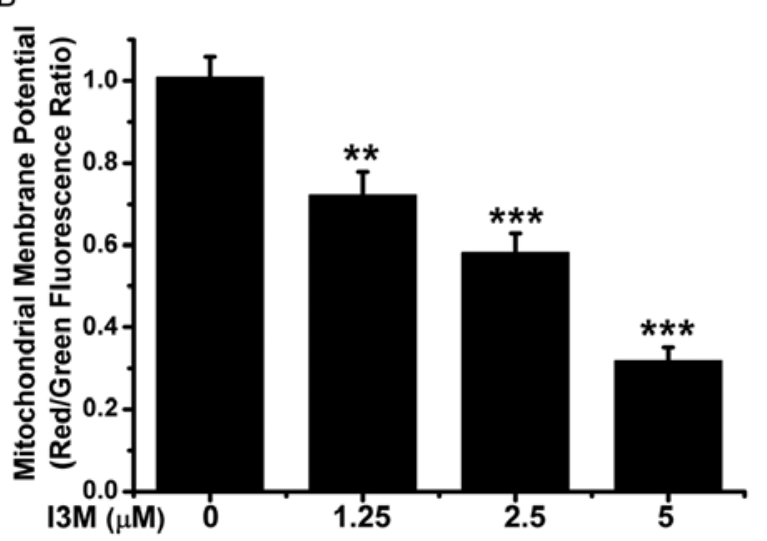

I3M $2.5 \mu \mathrm{M}$

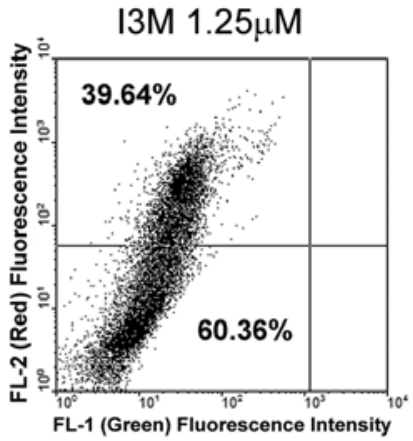

ity .
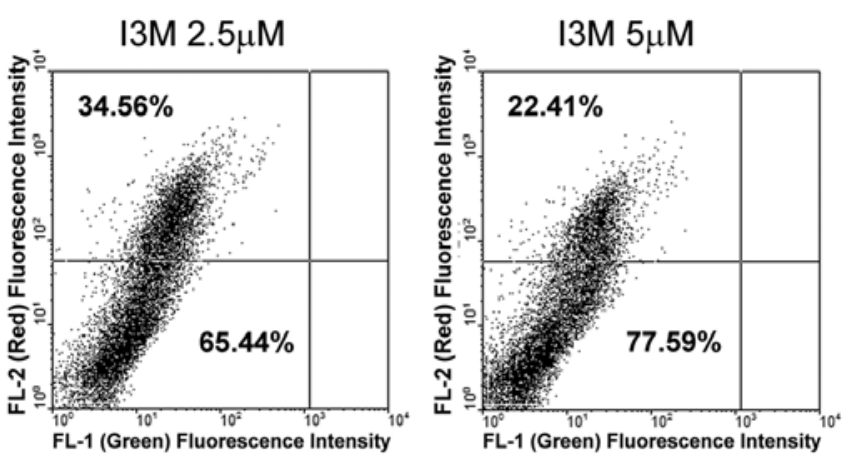

Figure 6. I3M decreases mitochondrial membrane potential $\left(\Delta \Psi_{\mathrm{m}}\right)$ in LA-N-1 cells. LA-N-1 cells were seeded in 24 -well plates at a density of $1.5 \times 10^{5}$ cells per well. On the following day, cells were treated with solvent control (0.1\% DMSO) or I3M at the indicated concentrations for $24 \mathrm{~h} . \Delta \Psi_{\mathrm{m}}$ was determined by flow cytometry with the dye JC-1. Cells that emit more red fluorescence indicate higher membrane potential. (A) The numbers at the corners represent the percentage of cells in the corresponding quadrants. Results are representative of three independent experiments. (B) The red (FL-2) to green (FL-1) fluorescence ratios for LA-N-1 cells treated with various concentrations of I3M for $24 \mathrm{~h}$ are shown. ${ }^{* *} \mathrm{P}<0.01 ;{ }^{* * *} \mathrm{P}<0.001$.
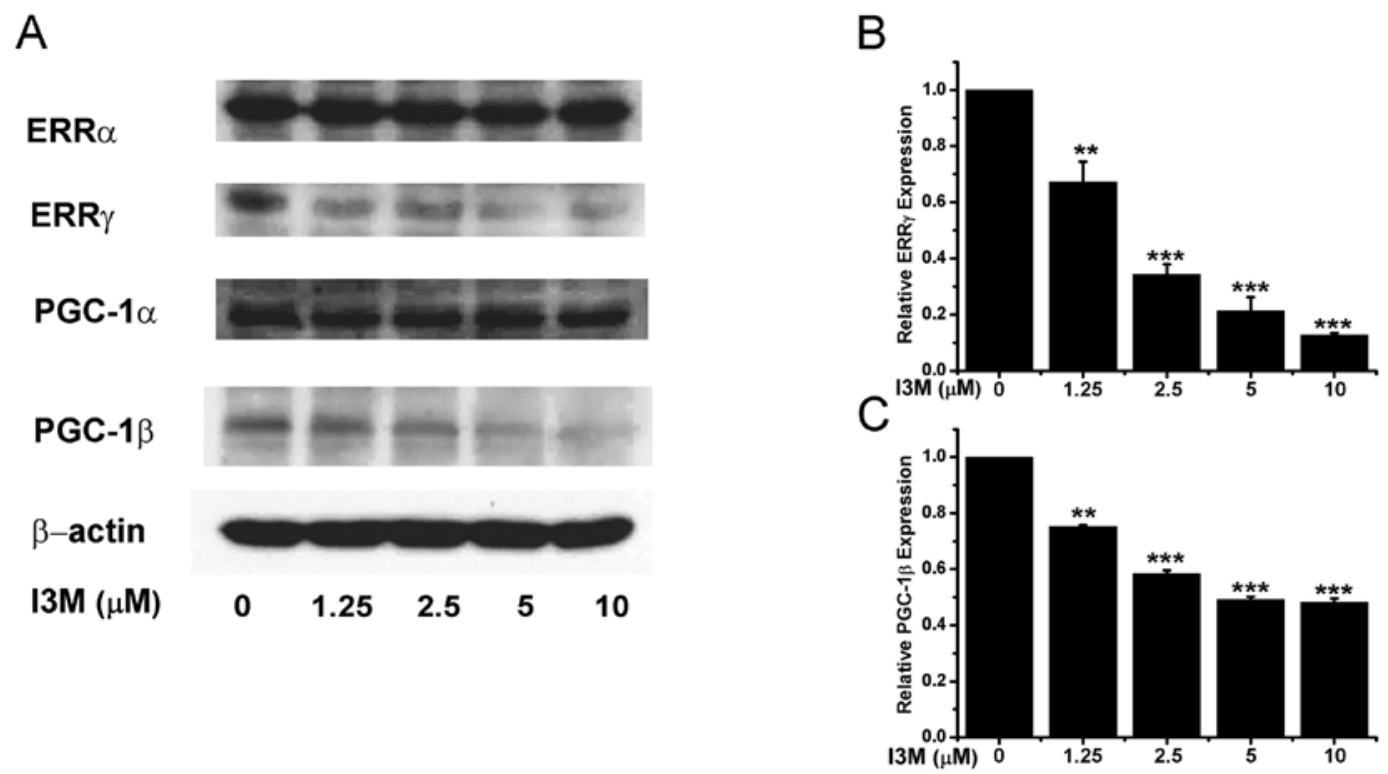

Figure 7. I3M downregulates the expression of ERR $\gamma$ and PGC-1 $\beta$. (A) Representative western blots of ERR $\alpha$, ERR $\gamma$, PGC- $1 \alpha$, PGC-1 $\beta$, with $\beta$-actin as a control after treatment with I3M for $48 \mathrm{~h}$ in LA-N-1 cells. The mRNA expression levels of ERR $\gamma(\mathrm{B})$ and PGC-1 $\beta$ (C) were measured after $24 \mathrm{~h}$ of I3M treatment in LA-N-1 cells. The relative level for the solvent-treated control was set as $1 .{ }^{* *} \mathrm{P}<0.01 ;{ }^{* * *} \mathrm{P}<0.001$.

Indirubin-3'-oxime selectively reduces ERR $\gamma$ and $P G C-1 \beta$ protein and $m R N A$ levels in $L A-N-1$ cells. Our previous results showed that I3M suppressed the mRNA expression levels of SOD2, Tfam and ATP5b in LA-N-1 cells. It has been reported that ERR $\alpha, E R R \gamma$, PGC-1 $\alpha$, and PGC- $1 \beta$ are responsible for controlling the expression of these genes at the transcriptional level and regulate mitochondrial mass and oxidative phosphorylation (17). Therefore, whether I3M can alter the mRNA and protein levels of these transcriptional factors and co-activators was investigated. Using western blot analysis, the protein levels of ERR $\alpha$ and PGC- $1 \alpha$ were not found to be significantly altered by I3M (Fig. 7A). On the contrary, the protein levels of ERR $\gamma$ and PGC-1 $\beta$ were markedly reduced (Fig. 7A). To determine whether the suppression was at the transcriptional level, the mRNA levels of ERR $\gamma$ and PGC- $1 \beta$ were determined by quantitative RT-PCR analysis. The mRNA expression levels 
of ERR $\gamma$ and PGC-1 $1 \beta$ were significantly decreased after I3M treatment (Fig. 7B and C).

\section{Discussion}

Neuroblastoma is a childhood cancer that, despite intensive multimodality therapy, is often fatal. Conventional treatments often cause severe and inevitable side-effects, therefore, there is a need for the development of alternative therapeutic approaches which are potent in inhibiting the proliferation of cancer cells while exhibiting minimal cytotoxicity towards the normal cells. Indirubin and its derivatives have been shown to have growth-inhibitory effects on various human cancer cells by inducing cell cycle arrest and/or apoptosis $(6,9-11,26-28)$. Previously, indirubin-3'-oxime, a derivative of indirubin, was shown to induce G0/G1 cell cycle arrest and apoptosis in Hep-2 human laryngeal carcinoma cells through induction of CDK inhibitor p21, inhibition of cyclin D1 and activation of caspase-3 (26). However, its modulatory effects on human neuroblastomas and the underlying action mechanisms remain poorly understood. In this study, the growth-inhibitory activities of I3M on the human neuroblastoma cells were investigated and the possible antitumor mechanisms were elucidated.

The effects of I3M on three human neuroblastoma cell lines, LA-N-1, SH-SY5Y and SK-N-DZ, were studied. Our results showed that treatment with I3M led to growth inhibition in all three human neuroblastoma cell lines in a dose- and time-dependent manner. The growth-inhibitory effect of I3M was most potent in LA-N-1 cells with the lowest $\mathrm{IC}_{50}$ value compared with those values for SH-SY5Y and SK-N-DZ cells. Therefore, LA-N-1 cells were selected for further mechanistic studies for the antitumor activity of I3M on human neuroblastoma cells. Markedly, I3M was found to exhibit little, if any, direct cytotoxicity on the normal cell models such as primary rat embryonic cortical neuronal cells, human embryonic kidney HEK-293 cells and human hepatocyte-like WRL-68 cells, at concentrations that are inhibitory to the neuroblastoma LA-N-1 cells, suggesting that I3M may be of therapeutic potential.

Cell growth is regulated by several factors, including growth factors, amino acids, and energy status to ensure that cell growth is appropriate to environmental conditions. In order to promote cell growth and division, cells need to coordinate synthesis of proteins, nucleic acids, and lipids for genome and organelle duplications. These processes consume high levels of energy. The mitochondrion is the cell's powerhouse responsible for energy production. Mitochondrial biogenesis and function play critical roles in cell growth and proliferation. For example, overexpression of a yeast homolog of mammalian Tfam shortens the G1 phase of the cell cycle (29). Downregulation of hMTERF4 inhibited cell proliferation, resulting in cell cycle arrest at the sub-G1 phase (14).

In this study, we demonstrated that I3M could block the human neuroblastoma cells' entry into the rapidly growing phase and this might be mediated, at least in part, by inducing mitochondrial dysfunction. We found that $\mathrm{I} 3 \mathrm{M}$ selectively reduced the expressions of ERR $\gamma$ and PGC-1 $1 \beta$, which are regulators of mitochondrial biogenesis and function. Downregulation of ERR $\gamma$ and PGC-1 $1 \beta$ is likely to be a primary event preceding some of the mitochondrial dysfunctions observed with I3M. In particular, the expression levels of Tfam, ATP5b and SOD2, which are regulated by PGC- $1 \alpha$, PGC- $1 \beta$ with ERR $\alpha$ or ERR $\gamma$ (17), were suppressed by I3M. Suppressing the expression of the oxidative phosphorylation enzyme (ATP5b) and mitochondrial biogenesis regulator (Tfam) is likely to result in reduced oxidative phosphorylation and the ability to generate ATP, which may be the main mechanism behind the decreased $\Delta \Psi_{\mathrm{m}}$ and cellular ATP levels, as previously reported (30). The elevation of ROS levels and reduction in ATP levels might be the reasons for the increased CDK inhibitor $\mathrm{p} 27^{\mathrm{Kip} 1}$, and decreased CDK2 and cyclin E levels, and cell cycle arrest of LA-N-1 cells at the $\mathrm{G0} / \mathrm{G} 1$ phase, and our results are in line with a previous report showing that the mitochondrion has a direct and specific role in the regulation of cell cycle progression (15).

Collectively, our findings indicate that I3M might exert its growth-inhibitory effect on the human neuroblastoma LA-N-1 cells by causing mitochondrial dysfunction which results in cell cycle arrest. Moreover, I3M exhibited minimal cytotoxicity towards the normal cells. Therefore, further elucidation of the action mechanisms of I3M on human neuroblastoma cells may provide better insights in the development of I3M as a drug candidate for the treatment of this pediatric cancer.

\section{Acknowledgements}

We thank Professor K.F. Lau for providing us with the primary embryonic cortical neurons from SD rats and Miss Ada Kong for her technical assistance in this study.

\section{References}

1. Maris JM: Recent advances in neuroblastoma. N Engl J Med 362: 2202-2211, 2010.

2. Park JR, Eggert A and Caron H: Neuroblastoma: biology, prognosis, and treatment. Hematol Oncol Clin North Am 24: 65-86, 2010.

3. Xiao Z, Hao Y, Liu B and Qian L: Indirubin and meisoindigo in the treatment of chronic myelogenous leukemia in China. Leuk Lymphoma 43: 1763-1768, 2002.

4 Zhang N, Jiang Y, Zou J, et al: 3D QSAR for GSK-3beta inhibition by indirubin analogues. Eur J Med Chem 41: 373-378, 2006.

5. Meijer L, Skaltsounis AL, Magiatis P, et al: GSK-3-selective inhibitors derived from Tyrian purple indirubins. Chem Biol 10: 1255-1266, 2003.

6. Hoessel R, Leclerc S, Endicott JA, et al: Indirubin, the active constituent of a Chinese antileukaemia medicine, inhibits cyclindependent kinases. Nat Cell Biol 1: 60-67, 1999.

7. Leclerc S, Garnier M, Hoessel R, et al: Indirubins inhibit glycogen synthase kinase-3 beta and CDK5/p25, two protein kinases involved in abnormal tau phosphorylation in Alzheimer's disease. A property common to most cyclin-dependent kinase inhibitors? J Biol Chem 276: 251-260, 2001.

8. Nguyen MC, Bui HT, Dang HH, et al: Inhibitory effects of indirubin derivatives on the growth of HL-60 leukemia cells. Nat Prod Commun 5: 103-106, 2010.

9. Damiens E, Baratte B, Marie D, Eisenbrand G and Meijer L: Anti-mitotic properties of indirubin-3'-monoxime, a CDK/GSK-3 inhibitor: induction of endoreplication following prophase arrest. Oncogene 20: 3786-3797, 2001.

10. Shi J and Shen HM: Critical role of Bid and Bax in indirubin3'-monoxime- induced apoptosis in human cancer cells. Biochem Pharmacol 75: 1729-1742, 2008.

11. Perabo FG, Landwehrs G, Frossler C, Schmidt DH and Mueller SC: Antiproliferative and apoptosis inducing effects of indirubin-3'-monoxime in renal cell cancer cells. Urol Oncol 29: 815-820, 2011.

12. Ravichandran K, Pal A and Ravichandran R: Effect of indirubin3-monoxime against lung cancer as evaluated by histological and transmission electron microscopic studies. Microsc Res Tech 73: 1053-1058, 2010. 
13. McBride HM, Neuspiel M and Wasiak S: Mitochondria: more than just a powerhouse. Curr Biol 16: R551-R560, 2006.

14. Yu M, Dai J, Huang WW, et al: hMTERF4 knockdown in HeLa cells results in sub-G1 cell accumulation and cell death. Acta Biochim Biophys Sin 43: 372-379, 2011.

15. Mandal S, Guptan P, Owusu-Ansah E and Banerjee U: Mitochondrial regulation of cell cycle progression during development as revealed by the tenured mutation in Drosophila. Dev Cell 9: 843-854, 2005

16. Owusu-Ansah E, Yavari A, Mandal S and Banerjee U: Distinct mitochondrial retrograde signals control the G1-S cell cycle checkpoint. Nat Genet 40: 356-361, 2008.

17. Giguere V: Transcriptional control of energy homeostasis by the estrogen-related receptors. Endocr Rev 29: 677-696, 2008.

18. Wu ZD, Puigserver P, Andersson U, et al: Mechanisms controlling mitochondrial biogenesis and respiration through the thermogenic coactivator PGC-1. Cell 98: 115-124, 1999.

19. Lo FH, Mak NK and Leung KN: Studies on the anti-tumor activities of the soy isoflavone daidzein on murine neuroblastoma cells. Biomed Pharmacother 61: 591-595, 2007.

20. Liu T, Hannafon B, Gill L, Kelly W and Benbrook D: Flex-Hets differentially induce apoptosis in cancer over normal cells by directly targeting mitochondria. Mol Cancer Ther 6: 1814-1822, 2007.

21. Opel D, Naumann I, Schneider M, Bertele D, Debatin KM and Fulda S: Targeting aberrant PI3K/Akt activation by PI103 restores sensitivity to TRAIL-induced apoptosis in neuroblastoma. Clin Cancer Res 17: 3233-3247, 2011.

22. Liao XM, Wang YF and Wong CW: Troglitazone induces cytotoxicity in part by promoting the degradation of peroxisome proliferator-activated receptor gamma co-activator-1 alpha protein. Br J Pharmacol 161: 771-781, 2010.
23. Freedman VH and Shin SI: Cellular tumorigenicity in nude mice: correlation with cell growth in semi-solid medium. Cell 3: 355-359, 1974.

24. Bianco MR, Berbenni M, Amara F, et al: Cross-talk between cell cycle induction and mitochondrial dysfunction during oxidative stress and nerve growth factor withdrawal in differentiated PC12 cells. J Neurosci Res 89: 1302-1315, 2011.

25. Zelko IN, Mariani TJ and Folz RJ: Superoxide dismutase multigene family: a comparison of the CuZn-SOD (SOD1), Mn-SOD (SOD2), and EC-SOD (SOD3) gene structures, evolution, and expression. Free Radic Biol Med 33: 337-349, 2002.

26. Kameswaran TR and Ramanibai R: Indirubin-3-monooxime induced cell cycle arrest and apoptosis in Hep-2 human laryngeal carcinoma cells. Biochem Pharmacol 63: 146-154, 2009.

27. Xingi E, Smirlis D, Myrianthopoulos V, et al: 6-Br-5methylindirubin-3'-oxime (5-Me-6-BIO) targeting the leishmanial glycogen synthase kinase-3 (GSK-3) short form affects cell-cycle progression and induces apoptosis-like death: exploitation of GSK-3 for treating leishmaniasis. Int J Parasitol 39: 1289-1303, 2009

28. Ribas J, Bettayeb K, Ferandin Y, et al: 7-bromoindirubin-3 -oxime induces caspase-independent cell death. Oncogene 25: 6304-6318, 2006.

29. Loewith R, Jacinto E, Wullschleger S, et al: Two TOR complexes, only one of which is rapamycin sensitive, have distinct roles in cell growth control. Mol Cell 10: 457-468, 2002.

30. Nadanaciva S, Dykens JA, Bernal A, Capaldi RA and Will Y: Mitochondrial impairment by PPAR agonists and statins identified via immunocaptured OXPHOS complex activities and respiration. Toxicol Appl Pharmacol 223: 277-287, 2007. 